\title{
Observer-Based Fault-Tolerant Predictive Control for LPV Systems with Sensor Faults: An Active Car Suspension Application
}

\author{
Abdelaziz Abboudi ${ }^{1}$, Sofiane Bououden ${ }^{2, *}$, Mohammed Chadli ${ }^{3}$, Ilyes Boulkaibet ${ }^{4}$ and Bilel Neji ${ }^{4}$ \\ 1 Laboratory of ISMA, Department of Mechanical Engineering, Faculty of Sciences and Technology, \\ Abbes Laghrour University, Khenchela 40004, Algeria; aboudist@univ-khenchela.dz \\ 2 Laboratory of SATIT, Department of Industrial Engineering, Abbes Laghrour University, \\ Khenchela 40004, Algeria \\ 3 IBISC, Université Paris-Saclay, Univ Evry, 91020 Evry, France; mohammed.chadli@univ-evry.fr \\ 4 College of Engineering and Technology, American University of the Middle East, Egaila 54200, Kuwait; \\ Ilyes.boulkaibet@aum.edu.kw (I.B.); bilel.neji@aum.edu.kw (B.N.) \\ * Correspondence: sofiane.bououden@univ-khenchela.dz
}

Citation: Abboudi, A.; Bououden, S.; Chadli, M.; Boulkaibet, I.; Neji, B. Observer-Based Fault-Tolerant Predictive Control for LPV Systems with Sensor Faults: An Active Car Suspension Application. Appl. Sci. 2022, 12, 684. https://doi.org/ 10.3390/app12020684

Academic Editor: Adel Razek

Received: 5 December 2021

Accepted: 8 January 2022

Published: 11 January 2022

Publisher's Note: MDPI stays neutral with regard to jurisdictional claims in published maps and institutional affiliations.

Copyright: (c) 2022 by the authors. Licensee MDPI, Basel, Switzerland. This article is an open access article distributed under the terms and conditions of the Creative Commons Attribution (CC BY) license (https:// creativecommons.org/licenses/by/ $4.0 /)$.

\begin{abstract}
In this paper, an observer-based robust fault-tolerant predictive control (ORFTPC) strategy is proposed for Linear Parameter-Varying (LPV) systems subject to input constraints and sensor failures. The main objective of this work is to establish a real observer based on a virtual observer to be used to estimate both states and sensor failures of the system. The proposed virtual observer is employed to improve the observation precision and reduce the impacts of the sensor faults and the external disturbances in the LPV systems. In addition, a real observer is proposed to overcome the virtual observer margins and to ensure that all states and sensor faults of the system are properly estimated, without the need for any fault isolation modules. The proposed solution demonstrates that, using both observers, a robust fault-tolerant predictive control is established via the Lyapunov function. Moreover, sufficient stability conditions are derived using the Lyapunov approach for the convergence of the proposed robust controller. Furthermore, the proposed approach simultaneously computes the gains of the real observer and the controller from a linear matrix inequality (LMI), which is deduced from the estimation errors. Finally, the performance of the proposed approach is investigated by a simulation example of a quarter-vehicle model, and the simulation results under a sensor fault illustrate the robustness and performance of the proposed method.
\end{abstract}

Keywords: linear parameter-varying systems (LPV); sensor faults; observers; tolerant predictive control; input constraints; active vehicle suspension

\section{Introduction}

Fault-Tolerant Control (FTC) systems are methods developed to deal with possible faults caused by sensors and/or actuators in terms of reducing the impacts of these faults and keeping the performance of the system acceptable. These approaches have the ability to maintain a performance close to the desirable performance while preserving stability conditions in the presence of several types of faults caused by sensors and/or actuators. Over the past several decades, several FTC approaches have been introduced and investigated in the literature, aiming to reduce the faults' impacts on the total stability and performance of systems [1-3].

Generally, there are two major classes of FTC methods: active (AFTC) and passive (PFTC). In the PFTC approach, the FTC system cannot respond in real-time to all fault events since the controller's structure and its parameters are formerly set and trained to only tolerate a specific set of faults [4]. As a result, a PFTC approach cannot adjust its parameters to deal with the occurrence of faults, in real-time, that are not within the predefined set. On the other hand, the AFTC methods are mainly developed to deal with the occurrence of faults by accommodating faults in such a way that the controller updates its parameters 
to reduce the faults' impacts on the control system [5,6]. As a result, the AFTC adjusts its structure or parameters based on the estimated faults obtained by the fault detection and identification (FDI) block, and the proposed AFTC design manipulates the estimated faults to maintain better performance in terms of stability, robustness, and fault tolerability [7].

Linear Parameter-Varying (LPV) modeling approaches have received considerable attention over the past three decades in modeling and control areas. This is due to their efficiency in dealing with tractable mathematical descriptions for relatively complex nonlinear systems. One of the main types of LPV systems is the polytopic form, which allows the system to be described as a form of a convex combination of sub-models, and these sub-models are defined by the vertices of a convex polytope. As a result, the sub-models are combined to form the LPV model of the system by convex weighing functions, resulting in the global model of the system. Many nonlinear systems have been successfully represented by LPV models [8-10].

Recently, many researchers have been attracted by the theory of solving nonlinear systems subject to faults based on the LPV representations, and many research papers have been published in the last few years. Li and Zhang [11] investigated robust H-1 filtering for singular LPV systems subject to time-varying delays, while the states of the system were estimated but without any FDI purposes. Hamdi et al. [12] proposed an approach to deal with polytopic models with unknown inputs and used a proportional integral observer for fault detection and isolation. Wang et al. [13] proposed and investigated an adaptive fault diagnosis observer-based approach for regular LTI systems to deal with actuator faults. Although this proposed approach can be easily modified to deal with LPV systems, unfortunately, the methodology itself can only detect and estimate constant actuator faults. Lira et al. [14] proposed a technique for LPV systems in which an observer-based approach is synthesized to estimate polymer electrolyte membrane (PEM) system states while varying parameters were planned with state variables. The proposed approach's performance was also tested for several common fault scenarios that may appear during normal PEM system operation. Yadigar et al. [15] proposed an approach based on a virtual actuator for fault-tolerant control of linear systems subject to time-varying additive actuator faults as well as an external disturbance. The proposed approach assumes that the structure of the nominal controller is known. Even though this approach was used to synthesize a controller for LTI systems, it can easily be modified to accommodate LPV systems. In [16,17], a H-infinity observer-based approach is designed to estimate states and sensor and/or actuator faults. Based on the synthesized observer, an output feedback fault-tolerant controller is established to guarantee the stability of a satellite attitude system. Recently, a novel observer-based approach for robust stable hybrid fault-tolerant predictive control was proposed by Zahaf et al. to investigate actuator faults in systems [18]. Recently, the virtual actuator and virtual sensor-based techniques have attracted more attention in dealing with different types of faults for LPV systems. The authors in [19] proposed an LPV virtual sensor design for FTC nonlinear systems, in which the nonlinear system is described as a LPV representation. In this proposed approach, the scheduling parameters for the controller, plant, and reconfiguration blocks are the same. In another approach proposed in [20], the input and the output matrices of the LPV model are set to have dependent parameters while different virtual actuator (VA) and virtual sensor (VS) parameter designs are allowed depending on whether the faults are total or partial. In addition, several practitioners have considered the implementation of a bank of reconfiguration blocks [1] technique in which each fault scenario is considered in the design. The proposed approach, based on predictive control, has shown good performance in handling actuator faults and maintaining robust trajectory tracking.

Generally, model predictive control (MPC) is considered to have been one of the most successful advanced control algorithms in many industrial applications over the past few decades [21-23]. However, the FTC problem has not been fully investigated for model predictive control, and the FTC-MPC topic has recently become attractive to many researchers, with several algorithms being proposed to deal with actuator faults. Zou et al. [24] proposed 
a model predictive fault-tolerant control (MPFTC) methodology optimized and adjusted by a Genetic algorithm. The proposed methodology was investigated for batch processes under disturbances and partial actuator faults. Zarch et al. [25] proposed an actuator fault-tolerance controller based on nonlinear model predictive control systems. In this approach, the viability theory is used to synthesize the controller. Shi et al. [26] proposed a robust constrained model predictive fault-tolerant control methodology for systems subject to uncertainties, unknown disturbances, interval time-varying delays, and partial actuator failures. Similarly, Sheikhbahaei et al. [27] proposed a new methodology for explicit model predictive control of linear discrete-time systems under linear constraints and subject to unknown disturbances, uncertainties, and actuator faults. Zhang et al. [28] proposed and presented a state-space model predictive fault-tolerant control methodology for batch processes subject to unknown disturbances and partial actuator faults. It is worth mentioning that most of the proposed approaches based on MPC algorithms for nonlinear discrete-time systems are based on the linearization of systems around operating points, which makes these approaches less accurate compared with nonlinear methodologies. In addition, it can be clearly observed that FTC-based MPC problems have not been fully investigated for LPV discrete-time systems subject to input constraints and sensor/actuator faults.

In this paper, an approach based on a virtual observer is proposed for discrete-time LPV systems subject to sensor faults. The proposed LPV model is used to simplify the complexity of the nonlinear system for the fault-tolerant predictive control (FTPC) and, at the same time, to maintain high accuracy for the controlling procedure. The proposed virtual observer is similar in theory to the observer presented in $[16,17]$ but incorporated with the MPC approach. As a result, an extended state-space LPV model is used to construct a virtual observer, then the formulated virtual observer is used to propose a real observer. Knowing that the real observer is based on the parameters of the virtual observer, both proposed observers will be used to formulate the fault-tolerant predictive control strategy. The proposed methodology has the ability to reduce the influences of faults in the dynamics of the system and ensure the robust stability of the closed-loop system. In addition, sufficient stability conditions are proposed, in terms of linear matrix inequality (LMI) constraints, to ensure the robust stability of the overall closed-loop system containing system states, and the estimation of error dynamics. Moreover, the gains of both observers and the fault-tolerant controller are computed by solving the linear matrix inequalities (LMIs). This paper is structured as follows: the formulation of the LPV as well as fault representations are presented in Section 2. Section 3 presents both virtual and real observers to estimate the states and sensor faults at the same time. Based on the LPV model, an augmented robust observer fault-tolerant predictive control strategy is presented in Section 4. The simulation results are illustrated in Section 5. Finally, Section 6 concludes the paper.

Notation. The superscripts $A^{T}$ and $A^{-1}$ are used to denote the transposition and inverse of matrix $A$, respectively. The symbols $F_{r}$ describe the controller gains matrices while the terms $L_{r}$ represent the observer gain matrices. $\mathbb{R}^{n}$ denotes the $n$th dimensional Euclidean space. $0_{n}$ and $I_{n}$ are the zero matrix and the identity matrix, respectively. In addition, the symbol $\|p\|$ represents the Euclidean norm of vector $p$. The term $R M S(\cdot)$ represents the root mean square function. The symbol * is used to describe the symmetric part in equations. The term $C o\{\cdot\}$ denotes a convex hull, where its element "." refers to the vertex of the polyhedron. In addition, the matrices $M_{r}, N_{r}, X_{r}, Y_{r}, Z_{r}$, and $H_{r}$ are symmetric positive definite matrices. The expression $S \geq 0$ (or $S>0$ ) denotes that the matrix $S$ is positive semi-definite (or positive definite, respectively). Finally, the symbols $Q_{0}, R_{0}$ are the weighting matrices. 


\section{Convex Polytopic Model}

Consider the following polytopic discrete-time model given by:

$$
\left\{\begin{array}{c}
x(k+1)=A\left(\theta_{k}\right) x(k)+B\left(\theta_{k}\right) u(k) \\
y(k)=C\left(\theta_{k}\right) x(k)
\end{array}\right.
$$

where $x(k) \in \mathbb{R}^{n_{x}}$ is the system state, $y(k) \in \mathbb{R}^{n_{y}}$ is the system output vector, and $u(k) \in \mathbb{R}^{n_{u}}$ is the system input vector. The system matrices can be written in the polytopic form as $A\left(\theta_{k}\right)=\sum_{r=1}^{N} \rho_{r}\left(\theta_{k}\right) A_{r}, B\left(\theta_{k}\right)=\sum_{r=1}^{N} \rho_{r}\left(\theta_{k}\right) B_{r}$, and $C\left(\theta_{k}\right)=\sum_{r=1}^{N} \rho_{r}\left(\theta_{k}\right) C_{r}$, where $\rho_{r}\left(\theta_{k}\right) \geq 0$ and $\sum_{r=1}^{N} \rho_{r}\left(\theta_{k}\right)=1$.

The parameters $\theta_{k}, k=1,2, \ldots, N$ are bounded in a predefined compact set $\Theta$, i.e., $\theta_{k} \in \Theta \forall k \in \mathbb{N}$.

Consequently, the possible values of $\theta_{k}$ are held within a polytope convex $\operatorname{Co}\left\{\rho_{1}, \ldots, \rho_{r}\right\}$ of $r=2^{m}$ vertices $\left\{\vartheta_{1}, \ldots, \vartheta_{r}\right\}$. The images of the matrix $A(\rho)$ for each vertex $\vartheta_{j}$ correspond to a set $\left\{\rho_{1}, \ldots, \rho_{r}\right\}$, while the components of the set $\left\{\rho_{1}, \ldots, \rho_{r}\right\}$ are known as the extreme of the polytope [15]. The system in Equation (1) can be represented by a convex interpolation of the vertices $\Theta$ and each vertex $\vartheta_{j}$ of the polytope, which is obvious because of the linear dependence on $\theta_{k}$.

Note that the control action is set in order to satisfy the constraints below:

$$
\|u(k)\|_{2} \leq u_{\max }
$$

where $u_{\max }$ is the maximum value (upper limit) of the control input.

Since the proposed polytopic discrete-time system is subject to both sensor faults and external disturbances, the real output signal measured by the sensor may be altered when a sensor fault occurs. The output of the system is then modeled as follows:

$$
y(k)=C\left(\theta_{k}\right) x(k)+f_{s}(k)
$$

where $f_{s} \in \mathbb{R}^{n_{y}}$ is the vector of additive sensor faults.

Throughout this work, the following Lemmas and assumptions are needed to derive the main results.

Lemma 1 (Schur complements lemma [29]). Given any real matrices $S, R$, and $Z$ with $S=S^{T}$ and $Z>0$, then

$$
S-R Z^{-1} R^{T}<0
$$

If and only if

$$
\left[\begin{array}{cc}
S & R^{T} \\
R & Z
\end{array}\right]<0
$$

Assumption 1. Sensor faults $f_{s}(k)$ and the external disturbance $\omega(k)$ are bounded, and there exists a positive scalar $\delta$ such that $\|\omega(k)\|<\delta$.

Remark 1. Note that Assumption 1 ensures that the increments of faults and the disturbances between two sampling time instants are bounded.

In this paper, only the sensor faults are considered and the model in Equation (1) can be rewritten as:

$$
\left\{\begin{array}{c}
x(k+1)=A\left(\theta_{k}\right) x(k)+B\left(\theta_{k}\right) u(k)+D\left(\theta_{k}\right) \omega(k) \\
y_{f}(k)=C x(k)+f_{s}(k)
\end{array}\right.
$$


where $u_{f} \in \mathbb{R}^{n_{u}}, x \in \mathbb{R}^{n_{x}}$, and $y_{f} \in \mathbb{R}^{n_{y}}$ denote the vector of the faulty control input, the vector of the faulty system state, and the faulty measurement output vector, respectively. Note that $f_{s} \in \mathbb{R}^{n_{y}}$ is assumed to be an additive sensor fault.

\section{Design of Observer-Based Control Law}

The main objective of this work is to propose a virtual observer to improve the estimations and provide better accuracy for both fault observation and the system's states.

First, an augmented system, which is proposed based on the system in Equation (4) and exposed to an external disturbance, is proposed for designing an observer that can estimate system state vector $x(k)$ and sensor faults $f_{s}(k)$. The augmented system is defined as:

$$
\left\{\begin{array}{c}
E_{1} \eta(k+1)=\mathcal{A}\left(\theta_{k}\right) \eta(k)+B\left(\theta_{k}\right) u(k)+D\left(\theta_{k}\right) \omega(k) \\
y_{f}(k)=E_{2} \eta(k)
\end{array}\right.
$$

where

$$
\eta=\left[\begin{array}{c}
x(k) \\
f_{s}(k)
\end{array}\right], \mathcal{A}\left(\theta_{k}\right)=\left[A\left(\theta_{k}\right) 0\right], E_{1}=[I 0], E_{2}=\left[C\left(\theta_{k}\right) I\right]
$$

Multiplying $T_{1}$ to both sides of (4), and by using fact (5), i.e., $T_{1} E_{1}+T_{2} E_{2}=I$, where $T_{1}=\left[\begin{array}{c}I_{n_{x}} \\ -C\end{array}\right]$ and $T_{2}=\left[\begin{array}{c}0_{n_{x} \times n_{y}} \\ I_{n_{y}}\end{array}\right]$, we have:

$$
\eta(k+1)=T_{1} \mathcal{A}\left(\theta_{k}\right) \eta(k)+T_{1} B\left(\theta_{k}\right) u(k)+T_{1} D \omega(k)+T_{2} E_{2} \eta(k+1)
$$

Let us define the following virtual observer:

$$
\hat{\eta}(k+1)=T_{1} \mathcal{A}\left(\theta_{k}\right) \hat{\eta}(k)+T_{1} B\left(\theta_{k}\right) u(k)+T_{1} D\left(\theta_{k}\right) \omega(k)+T_{2} E_{2} \eta(k+1)+L\left(\theta_{k}\right)\left(y_{f}(k)-E_{2} \hat{\eta}(k)\right)
$$

where $\hat{\eta}=\left[\begin{array}{l}\hat{x}(k) \\ \hat{f}_{s}(k)\end{array}\right]$ is the estimation of the system state $x(k)$ and sensor fault signals $f_{s}(k)$, and $L\left(\theta_{k}\right)$ is the observer gains, which will be obtained later.

To compute these gains, we will consider an augmented system with estimation error $e(k)$ of the observer in Equation (7) as $e(k)=\eta(k)-\hat{\eta}(k)$. Subtracting Equation (7) from Equation (6), the error dynamic equation is obtained as

$$
\begin{aligned}
e(k+1) & =\left(T_{1} \mathcal{A}-L E_{2}\right) e+T_{1} D \omega(k) \\
& =\left(T_{1} \mathcal{A}\left(\theta_{k}\right)-L\left(\theta_{k}\right) E_{2}\right) e+T_{1} D \omega(k)
\end{aligned}
$$

Remark 2. The virtual observer cannot be realized since it includes unreachable information, i.e., $T_{2} E_{2} \eta(k+1)$. Consequently, a real observer based on the virtual observer's information is synthesized by eliminating the unknown terms.

Therefore, we define the auxiliary variable $\mathcal{V}(k)=\hat{\eta}(k)-T_{2} E_{2} \eta(k+1)$. The real observer for the system in Equation (4) can be obtained by:

$$
\begin{gathered}
\mathcal{V}(k+1)=\overline{\mathcal{A}}\left(\theta_{k}\right) \mathcal{V}(k)+\overline{\mathcal{B}}\left(\theta_{k}\right) u(k)+\mathcal{L}\left(\theta_{k}\right) \hat{\eta}(k) \\
\hat{\eta}(k)=\mathcal{V}(k)+\overline{\mathcal{C}}\left(\theta_{k}\right) y_{f}(k)
\end{gathered}
$$

where a new auxiliary variable, $\mathcal{V}(k)$, is introduced along with the matrices $\overline{\mathcal{A}}\left(\theta_{k}\right), \overline{\mathcal{B}}\left(\theta_{k}\right)$, and $\overline{\mathcal{C}}\left(\theta_{k}\right)$, and the gain matrices $\mathcal{L}\left(\theta_{k}\right)$ are the observer matrices to be computed later as follows: 


$$
\begin{gathered}
\overline{\mathcal{A}}\left(\theta_{k}\right)=T_{1} \mathcal{A}\left(\theta_{k}\right)-L\left(\theta_{k}\right) E_{2}, \overline{\mathcal{B}}\left(\theta_{k}\right)=T_{1} B\left(\theta_{k}\right), \overline{\mathcal{C}}\left(\theta_{k}\right)=T_{2} E_{2} \text {, and } \\
\mathcal{L}\left(\theta_{k}\right)=L\left(\theta_{k}\right)+\left(T_{1} \mathcal{A}\left(\theta_{k}\right)-L\left(\theta_{k}\right) E_{2}\right) T_{2}
\end{gathered}
$$

where $L\left(\theta_{k}\right)$ is a time-varying observer gain matrix that depends on $\theta_{k}$, which is defined as

$$
L\left(\theta_{k}\right)=\sum_{r=1}^{N} \rho_{r}\left(\theta_{k}\right) L_{r}
$$

\section{Robust Fault-Tolerant Predictive Control}

Next, a fault-tolerant predictive controller based on an observer is designed for LPV systems subject to disturbances and sensor faults. As the initial part of this work, an observer was constructed to estimate faulty signals of the system subject to an external disturbance. Next, based on the observer information a fault-tolerant predictive controller is proposed to guarantee stability and maintain control performance.

Next, the control law in Equation (10) is considered for compensating for the impacts of the faults in the closed-loop system and that rejects the disturbance's influence.

$$
u(k)=F\left(\theta_{k}\right) E_{1} \hat{\eta}(k)
$$

where $F$ is a time-varying gain matrix that depends on $\theta_{k}$, and can be defined as $F\left(\theta_{k}\right)=\sum_{r=}^{N} \rho_{r}\left(\theta_{k}\right) F_{r}$

Replacing Equation (10) in Equation (4), we obtain:

$$
\begin{aligned}
x(k+1) & =A\left(\theta_{k}\right) x(k)+B\left(\theta_{k}\right)\left(F\left(\theta_{k}\right) x(k)-F\left(\theta_{k}\right) E_{1} e(k)\right)+D\left(\theta_{k}\right) \omega(k) \\
& =\left(A\left(\theta_{k}\right)+B\left(\theta_{k}\right) F\left(\theta_{k}\right)\right) x(k)-B\left(\theta_{k}\right) F\left(\theta_{k}\right) E_{1} e(k)+D\left(\theta_{k}\right) \omega(k)
\end{aligned}
$$

To obtain the predictive controller, an optimization problem that minimizes the following worst-case quadratic objective function in an infinite horizon is considered [30]:

$$
\underbrace{\min }_{u_{c}(k)} \max J_{\infty}(k)
$$

Subject to (6) and $\|u(k)\|_{2} \leq u_{\max }$, where

$$
J_{\infty}(k)=\sum_{i=0}^{\infty}\|x(k+i)\|_{Q}^{2}+\|u(k+i)\|_{R}^{2}+\alpha^{2}\|\omega(k)\|^{2}
$$

where $Q$ and $R$ are known positive-definite weighting matrices.

Next, sufficient stability conditions, in terms of LMIs, are derived to ensure the stability of the overall closed-loop system in Equation (5).

Theorem 1. Consider the faulty LPV system in Equation (5). The state-feedback controller given by Equation (4) will robustly stabilize the system in Equation (6) subjected to external disturbances and sensor faults if there exist symmetric positive definite matrices $M_{r}, N_{r}, X_{r}, Y_{r}, Z_{r}$, and $H_{r} r=1, \ldots, N$, and a positive scalar $\gamma$ that satisfies the following convex optimization problem:

$$
\min _{\gamma, M_{r}, N_{r}, X_{r}, Y_{r}, Z_{r}, H_{r}} \gamma
$$

Subject to

$$
\left[\begin{array}{ccc}
-1 & * & * \\
\bar{x}(k) & -M_{r} & * \\
e(k) & 0 & -N_{r}
\end{array}\right]
$$




$$
\begin{aligned}
& {\left[\begin{array}{cc}
-u_{\max }^{2} & Y_{r} \\
Y_{r}^{T} & M_{r}-X_{r}^{T}-X_{r}
\end{array}\right] \leq 0} \\
& {\left[\begin{array}{cccccccc}
-\Xi_{1} & * & * & * & * & * & * & * \\
R^{\frac{1}{2}} Y_{r} & -\gamma I & * & * & * & * & * & * \\
Q^{\frac{1}{2}} X_{r} & 0_{n} & -\gamma I & * & * & * & * & * \\
0 & 0_{n} & 0_{n} & -\frac{\alpha^{2} I}{\gamma} & * & * & * & * \\
0 & 0_{n} & 0_{n} & 0_{n} & -\Xi_{2} & * & * & * \\
-R^{\frac{1}{2}} Y_{r} E_{1} & 0_{n} & 0_{n} & 0_{n} & 0_{n} & -\gamma I & * & * \\
A_{r} X_{r}+B_{r} Y_{r} & -B_{r} Y_{r} E_{1} & D_{r} X_{r} & 0_{n} & 0_{n} & 0_{n} & -M_{j} & * \\
0_{n} & T_{1} Z_{r} \mathcal{A}_{r}-H_{r} E_{2} & Z_{r} T_{1} D_{r} & 0_{n} & 0_{n} & 0_{n} & 0_{n} & -N_{j}
\end{array}\right]<0} \\
& r, j=1, \ldots, N
\end{aligned}
$$

where $\Xi_{1}=-M_{r}+X_{r}^{T}+X_{r}$ and $\Xi_{2}=-N_{r}+X_{r}^{T}+X_{r}$.

Additionally, the control gain can be calculated by $F_{r}=Y_{r} X_{r}^{-1}$ and the gain of the state observer is obtained by $L_{r}=Z_{r}^{-1} H_{r}, r=1, \ldots, N$.

Proof of Theorem 1. First, the following Lyapunov function is defined to obtain the stability conditions:

$$
V(k / k)=x^{T}(k / k) \Gamma\left(\theta_{k}\right) x(k / k)+e^{T}(k / k) \Pi\left(\theta_{k}\right) e(k / k)
$$

where $\Gamma\left(\theta_{k}\right)=\sum_{r=1}^{N} \rho_{r}\left(\theta_{k}\right) \Gamma_{r}$ with $\Gamma_{r}=\gamma M_{r}^{-1}$, and $\Pi\left(\theta_{k}\right)=\sum_{r=1}^{N} \rho_{r}\left(\theta_{k}\right) \Pi_{r}$ with $\Pi_{r}=\gamma N_{r}^{-1}$. Furthermore, $\Gamma\left(\theta_{k+1}\right)=\sum_{j=1}^{N} \rho_{r}\left(\theta_{k+1}\right) \Gamma_{j}$ and $\Pi\left(\theta_{k+1}\right)=\sum_{j=1}^{N} \rho_{r}\left(\theta_{k+1}\right) \Pi_{j}$ and

$$
V(k+i / k)=x^{T}(k+i / k) \Gamma\left(\theta_{k}\right) x(k+i / k)+e^{T}(k+i / k) \Pi\left(\theta_{k}\right) e(k+i / k)
$$

For any $i \geq 0$, suppose $V(k+i / k)$ satisfies the following stability constraint:

$$
V(k+i+1 / k)-V(k+i / k) \leq-\left[\|x(k+i / k)\|_{Q}^{2}+\left\|u\left(k+\frac{i}{k}\right)\right\|_{R}^{2}\right]+\alpha^{2}\|\omega(k)\|^{2}
$$

As it is assumed that summation is up to $\infty$, i.e., $i \rightarrow \infty, x(\infty)=0$, summing from $i=0$ to $\infty$ produces:

$$
J_{\infty}(k) \leq V(k+i / k)
$$

By defining $V(k+i / k) \leq \gamma$, an upper bound on the performance index is obtained as $J_{\infty}(k) \leq \gamma$.

Hence, the first inequality of Equation (20) holds.

Next, we show that the second inequality of Equation (15) holds.

$$
x(k / k) \Gamma\left(\theta_{k}\right) x(k / k)+e(k / k) \Pi\left(\theta_{k}\right) e(k / k) \leq \gamma
$$

Since the inequality (18) implies that $V(\zeta(k+j+1 / k))$ strictly decreases as $j$ goes to $\infty$ and $V(\zeta(k / k)) \leq \gamma$ from (18), we have

$$
\frac{1}{\gamma} x(k / k) \Gamma\left(\theta_{k}\right) x(k / k)+\frac{1}{\gamma} e(k / k) \Pi\left(\theta_{k}\right) e(k / k) \leq 1
$$


by the Schur complement, we obtain

$$
\left[\begin{array}{ccc}
-1 & * & * \\
x(k) & -\gamma \Gamma\left(\theta_{k}\right)^{-1} & * \\
e(k) & 0 & -\gamma \Pi\left(\theta_{k}\right)^{-1}
\end{array}\right]
$$

Substituting $\Gamma_{r}=\gamma M_{r}^{-1}$ and $\Pi_{r}=\gamma N_{r}^{-1}$ into the above inequality, and by applying the congruence transformation to the resulting inequality with Diag $\left[1, M_{r}^{-1}, N_{r}^{-1}\right]$, we conclude that Equation (15) holds.

Consider the input constraint in Equation (2)

$$
\begin{gathered}
\|u(k+i / k)\|_{\max } \triangleq \max _{i} u_{i}(k+i / k) \\
\max _{i>0}\|u(k)\|_{\max }=\max _{i>0}\|F \hat{x}(k)\|_{\max } \\
\max _{i>0}\|u(k)\|_{\max } \leq\left\|F\left(\frac{1}{\gamma} \Gamma_{r}\right)^{\frac{1}{2}}\right\|_{2}^{2} \Leftrightarrow \\
u_{\max }^{2} \leq \Gamma_{r^{2}}^{\frac{1}{2}} F^{T} F \Gamma_{r}^{\frac{1}{2}} \\
-u_{\max }^{2}+\left(\frac{1}{\gamma} \Gamma_{r}\right)^{\frac{1}{2}} F^{T} F\left(\frac{1}{\gamma} \Gamma_{r}\right)^{\frac{1}{2}} \leq 0
\end{gathered}
$$

Using the Schur complement, we obtain:

$$
\left[\begin{array}{cc}
-u_{\max }^{2} & F \\
F^{T} & -\frac{1}{\gamma} \Gamma_{r}
\end{array}\right] \leq 0
$$

Substituting $\Gamma_{r}=\gamma M_{r}^{-1}$ and $F_{r}=Y_{r} X_{r}^{-1}$ followed by multiplying the right by $\left[\begin{array}{cc}I & 0 \\ 0 & X_{r}\end{array}\right]$ and the left by $\left[\begin{array}{cc}I & 0 \\ 0 & X_{r}^{T}\end{array}\right]$, we obtain (16).

Now, (17) implies that:

$$
\left[\begin{array}{cccccccc}
-\Xi_{1} & * & * & * & * & * & * & * \\
R^{\frac{1}{2}} Y_{r} & -\gamma I & * & * & * & * & * & * \\
Q^{\frac{1}{2}} X_{r} & 0_{n} & -\gamma I & * & * & * & * & * \\
0 & 0_{n} & 0_{n} & -\frac{\alpha^{2} I}{\gamma} & * & * & * & * \\
0 & 0_{n} & 0_{n} & 0_{n} & -\Xi_{2} & * & * & * \\
-R^{\frac{1}{2}} Y_{r} E_{1} & 0_{n} & 0_{n} & 0_{n} & 0_{n} & -\gamma I & * & * \\
A_{r} X_{r}+B_{r} Y_{r} & -B_{r} Y_{r} E_{1} & D_{r} X_{r} & 0_{n} & 0_{n} & 0_{n} & -X_{j} & * \\
0_{n} & T_{1} Z_{r} \mathcal{A}_{r}-H_{r} E_{2} & Z_{r} T_{1} D_{r} & 0_{n} & 0_{n} & 0_{n} & 0_{n} & -W_{j}
\end{array}\right]<0
$$

where $\Xi_{1}=-M_{r}+X_{r}^{T}+X_{r}, \Xi_{2}=-N_{r}+X_{r}^{T}+X_{r}$ and the matrices $M_{r}$ and $N_{r}$ are positives. Furthermore, since $X>0$, we have

$$
\left(X_{r}^{T}-M_{r}\right)^{T} X^{-1}\left(X_{r}-M_{r}\right) \geq 0 \Rightarrow-M_{r}+X_{r}^{T}+X_{r} \leq X_{r}^{T} M_{r}^{-1} X_{r}
$$

Consequently,

$$
\Rightarrow-N_{r}+X_{r}^{T}+X_{r} \leq X_{r}^{T} N_{r}^{-1} X_{r}
$$


By taking into account Equations (32) and (33), we can state that (17) implies

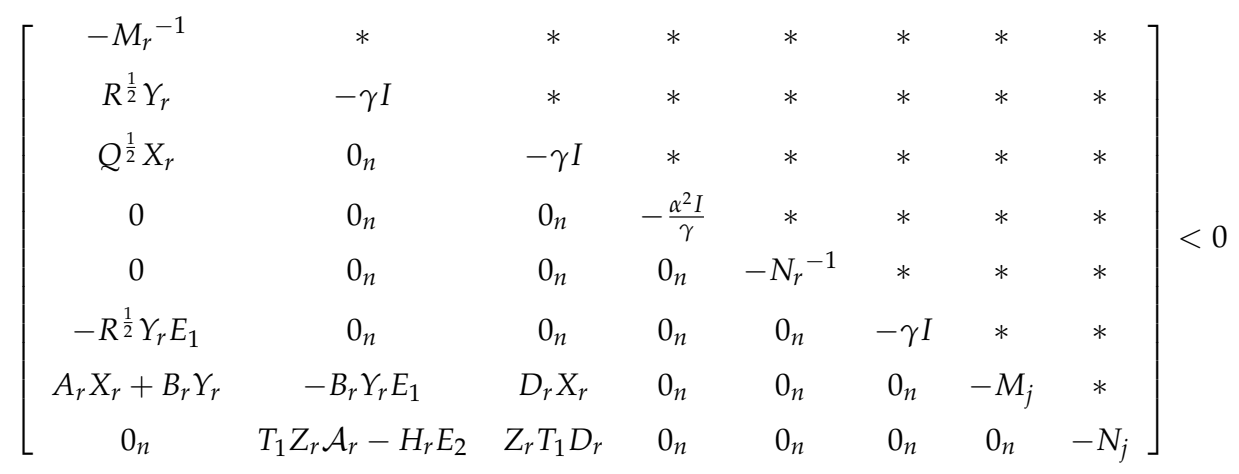

$$
r, j=1, \ldots, N
$$

where $\Gamma\left(\theta_{k}\right)=\sum_{r=1}^{N} \rho_{r}\left(\theta_{k}\right) \Gamma_{r}$ with $\Gamma_{r}=\gamma M_{r}^{-1}$, and $\Pi\left(\theta_{k}\right)=\sum_{r=1}^{N} \rho_{r}\left(\theta_{k}\right) \Pi_{r}$ with $\Pi_{r}=\gamma N_{r}^{-1}$. Furthermore, $\Gamma\left(\theta_{k+1}\right)=\sum_{j=1}^{N} \rho_{r}\left(\theta_{k+1}\right) \Gamma_{j}$ and $\Pi\left(\theta_{k+1}\right)=\sum_{j=1}^{N} \rho_{r}\left(\theta_{k+1}\right) \Pi$, and by substituting $Y_{r}=F_{r} X_{r}$ and $H_{r}=Z_{r} L_{r}$ into Equation (29), pre-multiplying and post-multiplying by Diag $\left[X_{r}^{-\mathrm{T}}, I, I, I, N_{r}^{-1}, I, I, I\right]$, and using the Schur complement, we see that this is equivalent to:

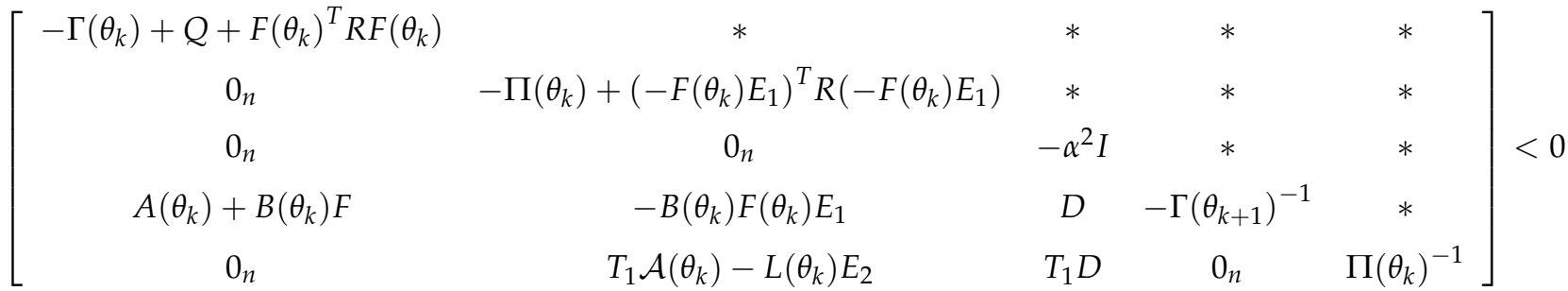

Again, applying the Schur complement to Equation (35):

$$
\begin{aligned}
& {\left[\begin{array}{cc}
A\left(\theta_{k}\right)+B\left(\theta_{k}\right) F\left(\theta_{k}\right) & 0 \\
-B\left(\theta_{k}\right) F\left(\theta_{k}\right) E_{1} & T_{1} \mathcal{A}-L E_{2} \\
D & T_{1} D
\end{array}\right]^{T}\left[\begin{array}{cc}
\Gamma\left(\theta_{k+1}\right) & 0 \\
0 & \Pi\left(\theta_{k+1}\right)
\end{array}\right]\left[\begin{array}{ccc}
A\left(\theta_{k}\right)+B\left(\theta_{k}\right) F & -B\left(\theta_{k}\right) F\left(\theta_{k}\right) E_{1} & D \\
0 & T_{1} \mathcal{A}\left(\theta_{k}\right)-L\left(\theta_{k}\right) E_{2} & T_{1} D
\end{array}\right]} \\
& +\left[\begin{array}{ccc}
-\Gamma\left(\theta_{k}\right)+Q+F\left(\theta_{k}\right)^{T} R F\left(\theta_{k}\right) & * & * \\
0_{n} & -\Pi\left(\theta_{k}\right)+\left(-F\left(\theta_{k}\right) E_{1}\right)^{T} R\left(-F\left(\theta_{k}\right) E_{1}\right) & * \\
0_{n} & 0_{n} & -\alpha^{2} I
\end{array}\right]<0
\end{aligned}
$$

Then, multiplying the resulting inequality from the left by $[x(k) e(k) \omega(k)]$ and from the right by $[x(k) e(k) \omega(k)]$ and taking into account Equations (2) and (3), we have:

$$
V(k+i+1 / k)-V(k+i / k) \leq-\left[\|x(k+i / k)\|_{Q}^{2}+\left\|u\left(k+\frac{i}{k}\right)\right\|_{R}^{2}\right]+\mu^{2}\|\omega(k)\|^{2}
$$

This will conclude the proof of Theorem 1.

Next, a closed-loop system is implemented as a polytopic observer-based fault-tolerant predictive controller for LPV systems subject to sensor faults. 


\section{Results and Discussion}

In this section, the proposed methodology is considered for a quarter-vehicle active suspension system and illustrated in Figure 1 [31,32]. The quarter-vehicle suspension model parameters used in this study are displayed in Table 1.

Table 1. The parameters of the quarter-vehicle suspension system.

\begin{tabular}{ccc}
\hline Parameters & Description & Numerical Value \\
\hline$m_{S}$ & The mass of the quarter body & $972(\mathrm{~g})$ \\
$m_{u s}$ & Unsprung mass & $113.6(\mathrm{~kg})$ \\
$k_{S}$ & Suspension stiffness coefficient & $42,719.6(\mathrm{~N} / \mathrm{m})$ \\
$k_{u s}$ & Tyre stiffness coefficient & $101,115(\mathrm{~N} / \mathrm{m})$ \\
$c_{S}$ & The damping ratio of the damper & $1095\left(\mathrm{~N}{ }^{*} / \mathrm{m}\right)$ \\
$c_{u s}$ & The damping ratio of the pneumatic tire & $14.6\left(\mathrm{~N}^{*} \mathrm{~s} / \mathrm{m}\right)$ \\
$N_{S}$ & The passive suspension stiffness & $4271.96\left(10 \%\right.$ of $\left.k_{s}\right)$ \\
\hline
\end{tabular}

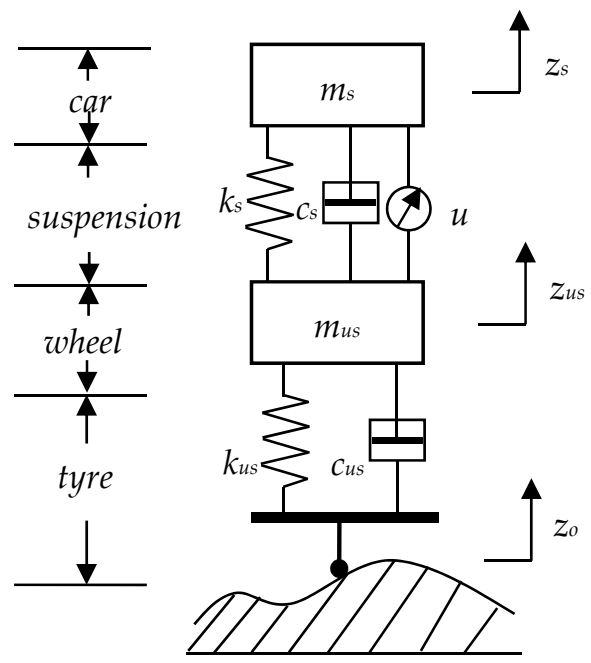

Figure 1. Nonlinear active quarter-car suspension model.

The mathematical model of the quarter-vehicle active suspension system is a nonlinear system described by the following nonlinear differential equations [27]:

$$
\begin{gathered}
m_{s} \ddot{z}_{s}(t)+c_{s}\left(\dot{z}_{s}(t)-\dot{z}_{u s}(t)\right)+k_{s}\left(z_{s}(t)-z_{u s}(t)\right)+N_{s}\left(z_{s}(t)-z_{u s}(t)\right)^{3}=-u(t) \\
m_{u s} \ddot{z}_{u s}(t)+c_{s}\left(\dot{z}_{u s}(t)-\dot{z}_{s}(t)\right)+k_{s}\left(z_{u s}(t)-z_{s}(t)\right)+N_{s}\left(z_{u s}(t)-z_{s}(t)\right)^{3} \\
+c_{u s}\left(\dot{z}_{u s}(t)-\dot{z}_{0}(t)\right)+k_{u s}\left(z_{u s}(t)-z_{0}(t)\right)=u(t)
\end{gathered}
$$

where $z_{u s}(t)$ and $z_{s}(t)$ represent the vertical displacements of the unsprung mass and the vertical displacements of the sprung mass, respectively. The vector $z_{o}(t)$ represents the road displacements. The active force (control signal) of the suspension system is given by $u(t)$. The sprung mass, which is also known as the car chassis, is given by $m_{s}$. The mass of the wheel assembly, also known as the unsprung mass, is given by $m_{u s}$. The parameters $k_{S}$ and $c_{S}$ are the spring coefficient and the damping coefficient of the suspension system, respectively. In addition, the parameters $k_{u s}$ and $c_{u s}$ are the compressibility and the damping of the pneumatic tire, respectively. Finally, $N_{S}$ is the nonlinear stiffness property of the primary suspension. In this study, more attention was paid to the vertical behavior of the tire, which is useful in the design and the analysis of the comfort of the passengers and the road handling of the vehicle. The tire can be modeled by a simple spring $k_{u s}$ that is in parallel with a damper $c_{u s}$. In addition, the stiffness of the spring is considered constant while its damping ratio is negligible. 
The nonlinear system described in Equation (32) can be modified to have the following state-space representation:

$$
\left\{\begin{array}{c}
x(k+1)=A\left(\theta_{k}\right) x(k)+B\left(\theta_{k}\right) u(k)+D\left(\theta_{k}\right) \omega(k) \\
y_{f}(k)=C\left(\theta_{k}\right) x(k)+f_{s}(k)
\end{array}\right.
$$

Note that the tire deflection is the state variable $x_{1}(t)=z_{u s}(t)-z_{0}(t)$. The rest of the state variables are the unsprung mass velocity $x_{2}(t)=\dot{z}_{u s}(t)$, the suspension stroke $x_{3}(t)=z_{\mathcal{S}}(t)-z_{u s}(t)$, and the sprung mass velocity $x_{4}(t)=\dot{z}_{\mathcal{S}}(t)$. The disturbance input that affects the system is represented as $\omega(t)=\dot{z}_{0}(t)$.

Note that the system matrix A can be expressed in polytopic form as:

$$
A\left(\theta_{k}\right)=\sum_{r=1}^{2} \rho_{r}\left(\theta_{k}\right) A_{r}
$$

where $\rho_{r}\left(\theta_{k}\right)$ are the vertex coefficients with a square signal $\alpha \in\left[\alpha_{1} \alpha_{2}\right]$ and $\rho_{1}=\left(\alpha_{2}-\alpha\right) /\left(\alpha_{2}-\alpha_{1}\right)$, $\rho_{2}=\left(\alpha-\alpha_{1}\right) /\left(\alpha_{2}-\alpha_{1}\right)$. The values of the vertex matrices $A\left(\theta_{k}\right)$ are $A_{1}=\left.A\right|_{\alpha=\alpha_{1}}$ and $A_{2}=\left.A\right|_{\alpha=\alpha_{2}}$. In this work, we assume that the suspension deflection cannot exceed a maximum value, and can be constrained by the mechanical structure $\left|z_{s}(t)-z_{u s}(t)\right|<\beta$. Note that the variable $\alpha(t)$ is given by the expression $\alpha(t)=\left(z_{s}(t)-z_{u s}(t)\right)^{2}=\left(x_{3}(t)\right)^{2}$. As a result, $x_{3}(t) \in[-\beta,+\beta]$ and $\alpha \in[0, \beta 2]$; that is, $\alpha_{1}=0$ and $\alpha_{2}=\beta^{2}$, and $\beta$ is a predefined constant.

Based on Equation (39), the faulty discrete state-space representation in Equation (38) can be expressed as a polytopic LPV system:

$$
\left\{\begin{array}{c}
x(k+1)=\sum_{r=1}^{2} \rho_{r}\left(\theta_{k}\right)\left(A_{r} x(k)+B_{r} u(k)+D_{r} \omega(k)\right) \\
y_{f}(k)=\sum_{r=1}^{2} \rho_{r}\left(\theta_{k}\right)\left(C_{r} x(k)+f_{s}(k)\right)
\end{array}\right.
$$

where the subscript $r$ refers to the corresponding vertex of the polytope, and $\rho_{r}\left(\theta_{k}\right)$ is the vertex coefficient subject to $\rho_{r}\left(\theta_{k}\right)>0$ and $\sum_{r=1}^{2} \rho_{r}\left(\theta_{k}\right)=1$.

Considering the LPV model as described in Equation (1) with $N=2$ vertices, the nonlinear system in Equation (31) is described by Equation (35) with the following matrices:

$$
\begin{gathered}
A_{1}=\left[\begin{array}{cccc}
0 & 1 & 0 & 0 \\
-\frac{k_{u s}}{m_{u s}} & \frac{c_{s}+c_{u s}}{m_{u s}} & \frac{k_{s}}{m_{u s}} & \frac{c_{s}}{m_{u s}} \\
0 & -1 & 0 & 1 \\
0 & \frac{c_{s}}{m_{s}} & -\frac{k_{s}}{m_{s}} & -\frac{c_{s}}{m_{s}}
\end{array}\right], A_{2}=\left[\begin{array}{cccc}
0 & 1 & 0 & 0 \\
-\frac{k_{u s}}{m_{u s}} & \frac{c_{s}+c_{u s}}{m_{u s}} & \frac{k_{s}-N_{s} \beta^{2}}{m_{u s}} & \frac{c_{s}}{m_{u s}} \\
0 & -1 & 0 & 1 \\
0 & \frac{c_{s}}{m_{s}} & -\frac{k_{s}+N_{s} \beta^{2}}{m_{s}} & -\frac{c_{s}}{m_{s}}
\end{array}\right] \\
B_{1}=B_{2}=\left[\begin{array}{c}
0 \\
\frac{1}{m_{u s}} \\
0 \\
-\frac{1}{m_{s}}
\end{array}\right], D_{1}=D_{2}=\left[\begin{array}{c}
-1 \\
\frac{c_{u s}}{m_{u s}} \\
0 \\
0
\end{array}\right] \text { with } \beta=0.2 .
\end{gathered}
$$

Next, the MPC parameters are selected as follows. The weight matrices $Q$ and $R$ of the cost function, in Equation (12), are set to $115 I$ and 15I, respectively, where $I$ is the identity matrix with a proper dimension. A constraint is set on the active control force to limit the power of the hydraulic actuator. Thus, a hard constraint is imposed on the active suspension such that $|u(k)| \leq u_{\max }$, and $u_{\max }=1500$. In addition, the initial state values of the LPV system are set as follows: $x(0)=[0,0,0,0]$ and for the augmented vector that includes initial fault $\eta(0)=[0,0,0,0,0.08]$. To test the robustness of the proposed method, the external disturbance, which is considered as a bump road profile, is expressed as $\omega(k)=0.05 \cos ($ pi $k) \sin (0.26$ pi $k)$. In the vehicle industry, the sensors are frequently 
subjected to strong vibrations that may add disturbances to the readings and cause sensor failure. Unfortunately, sensors with high vibration resistance are relatively expensive. In this study, we assumed that faults can be modeled in terms of vibration amplitude and frequency. So, the faults of the sensors injected into the closed-loop system are given by $f_{s}(k)=0.3(\cos 0.5 k+0.4)$. Note that suspension deflection is bounded and should not exceed a maximum value defined by the mechanical structure. The suspension deflection was bounded as $\left|z_{\mathcal{S}}(t)-z_{u \mathcal{S}}(t)\right|<z_{\max }$, where the maximum suspension deflection value $z_{\max }$ is $0.04 \mathrm{~m}$.

In this work, a LMI control toolbox in the MATLAB environment was used to solve the optimization problem in Equations (30)-(34). The results of the system states and their estimates and the fault with its estimate are illustrated in Figures 2-6, respectively.

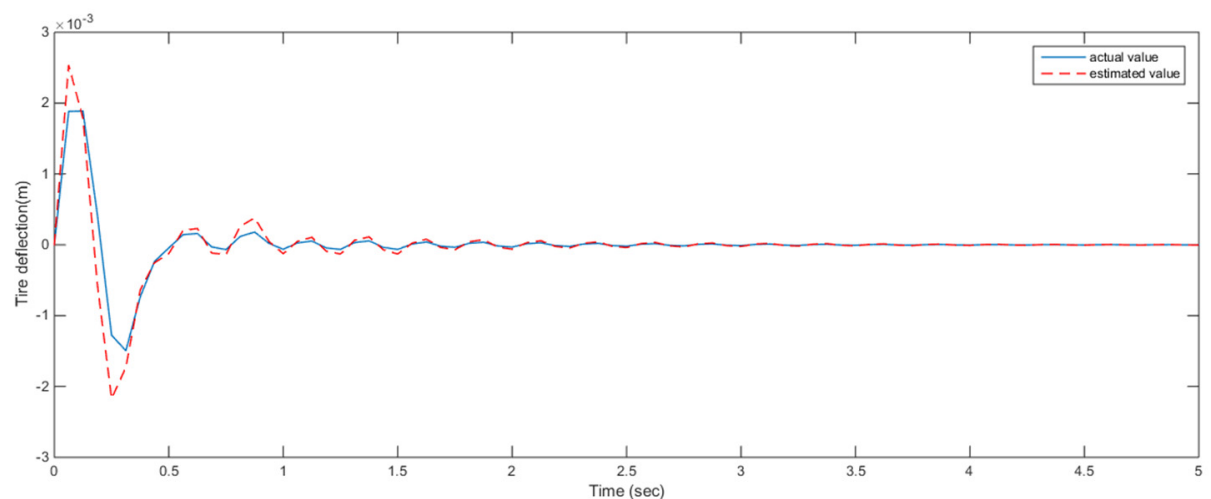

Figure 2. The actual and the estimated step response of tire deflection.

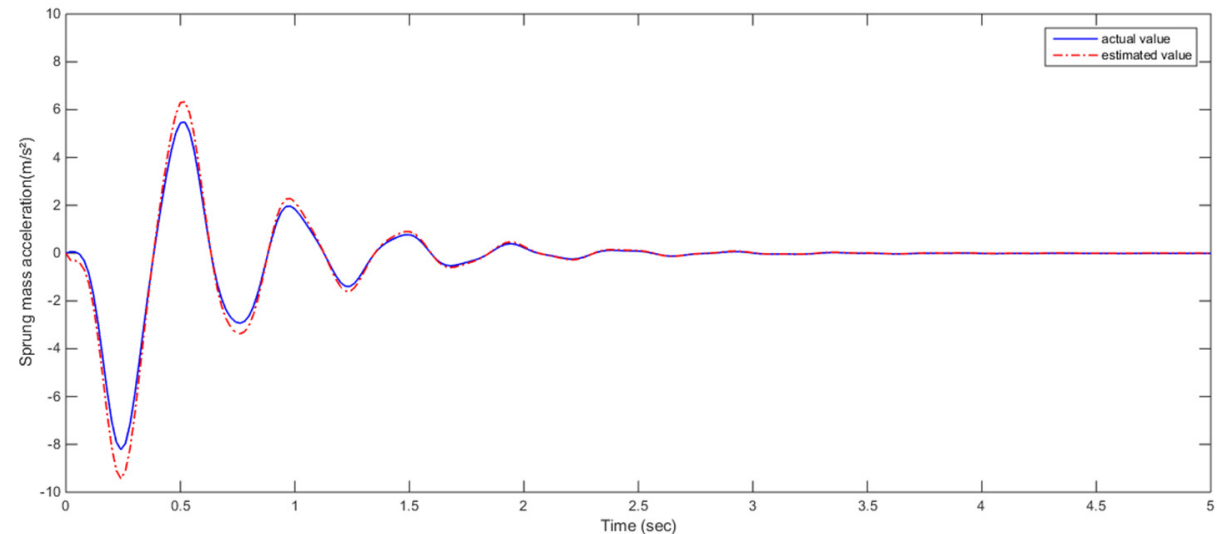

Figure 3. The step response of the actual and estimated sprung mass acceleration.

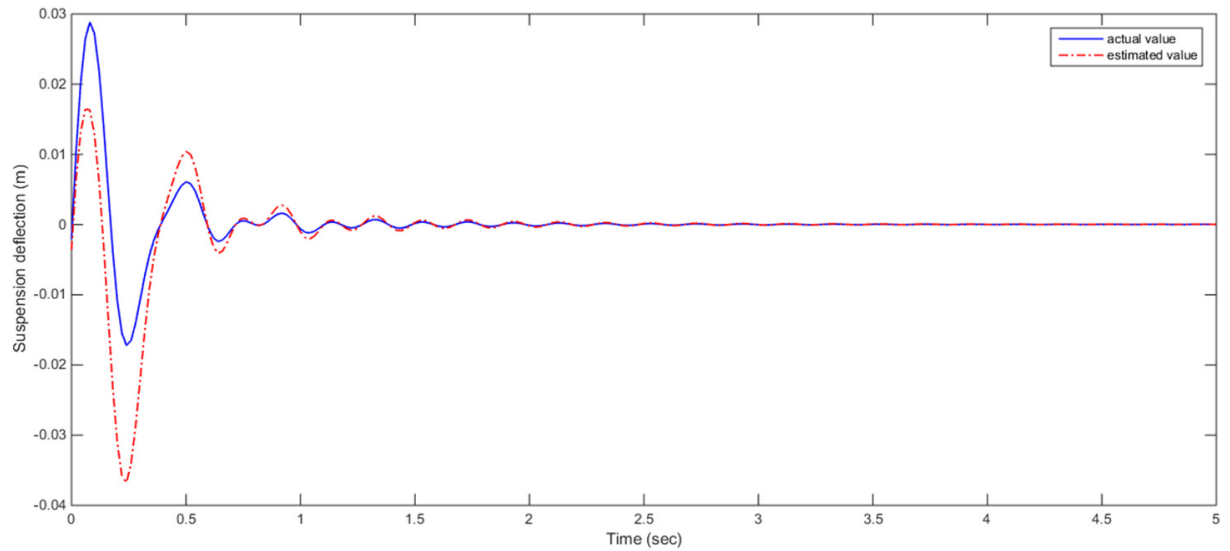

Figure 4. The step response of the actual and estimated suspension deflection. 


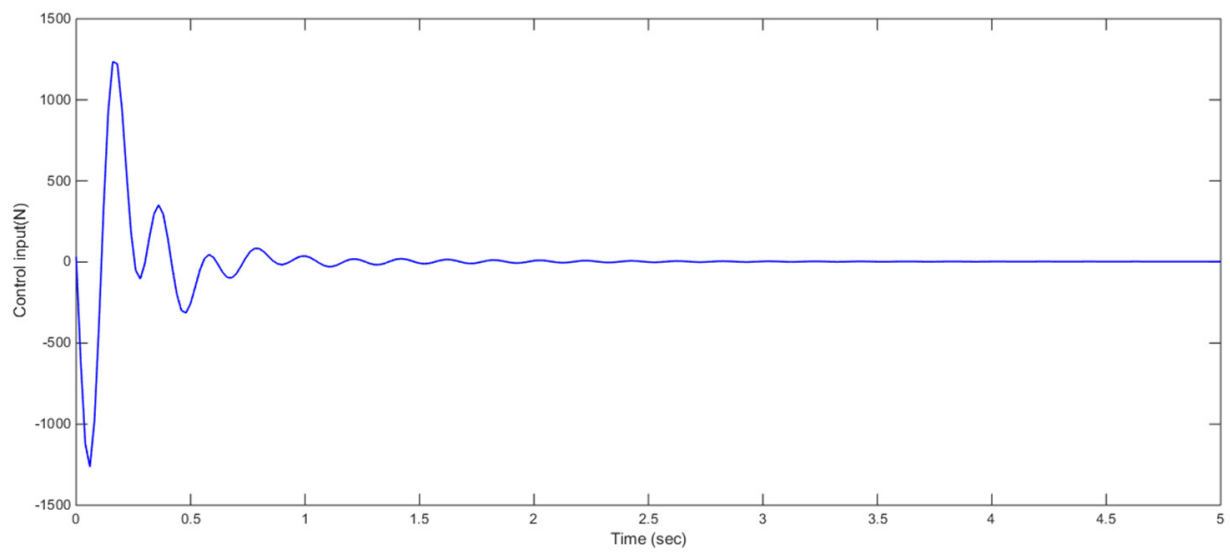

Figure 5. The control signal.

The results indicate that the proposed control methodology can robustly improve the ride comfort compared with passive FTC methods. The tire deflection and sprung mass acceleration responses together with their estimated values are plotted in Figures 2 and 3, respectively. The results in Figure 2 show that the tire deflection response has been considerably reduced despite the fact that the system is subject to real-time sensor faults and disturbances. The same comment can be made about the sprung mass acceleration results shown in Figure 3. In addition, the vertical acceleration of the vehicle's vibration, presented in Figure 3, is rapidly reduced by the fault-tolerant predictive control strategy while the actual value has been quickly and accurately estimated. As a result, the system may achieve lower values of body acceleration over time for the proposed active controller, which is better than the passive suspension systems proposed in [21] and, as a consequence, this will ensure better ride comfort.

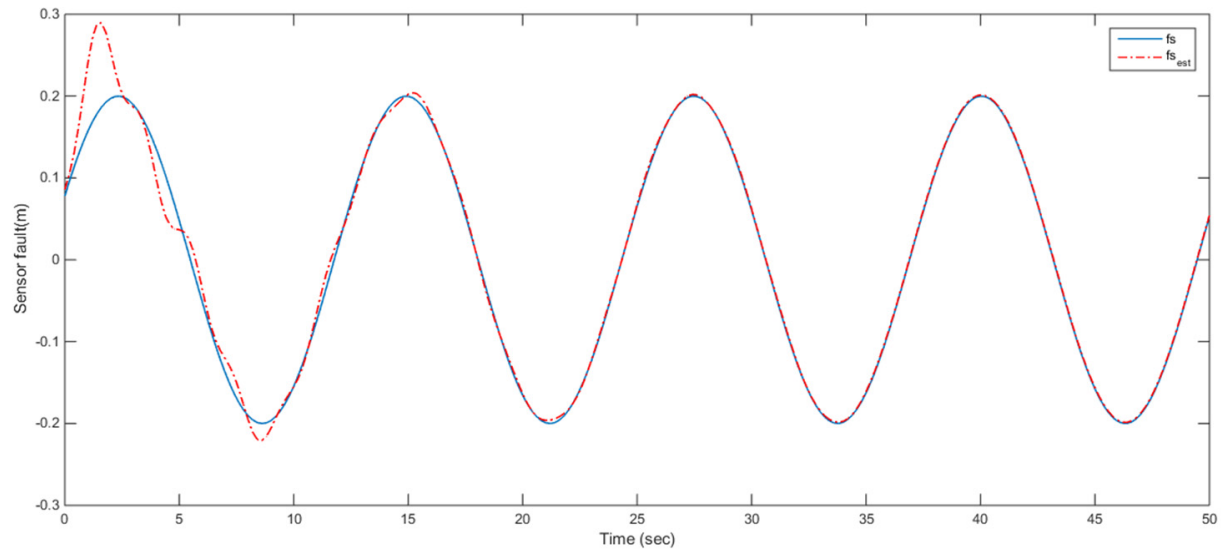

Figure 6. The proposed sensor fault and its estimation.

Figure 4 illustrates the suspension deflection, which is bounded by a maximum value of $0.04 \mathrm{~m}$, where the system achieves lower values over time for the closed-loop systems. In addition, the proposed methodology has reasonably estimated the suspension deflection values, and the values have never exceeded the maximum value, which is better than the results presented in [21].

Figure 5 illustrates the control signal $u(t)$, i.e., the actual force applied by the actuator. Note that the proposed robust predictive methodology considers the failures at the sensor and reacts by increasing the force needed. As a result, the proposed robust controller has improved the vehicle's ride comfort while satisfying all hard constraints, including both suspension deflection and actuator saturation constraints, even though the system is subject to an actuator fault. 
Figures 6 and 7 present the fault estimation results, which show that the stability of the closed-loop system is guaranteed under external disturbances and sensor faults. The estimation results show that the proposed methodology has excellent estimation performance and efficiently tolerates sensor faults.

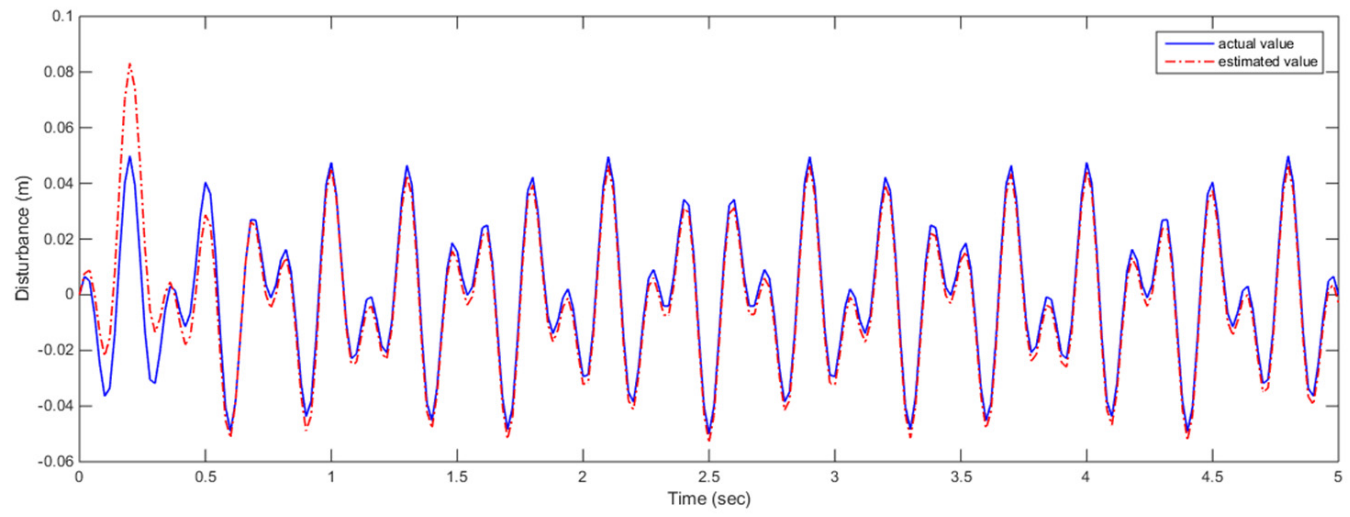

Figure 7. The disturbance and its estimation.

Table 2 summarises the RMS comparison between the proposed robust approach and the method discussed in [21]. The comparison describes the RMS errors of the vehicle's dynamic responses in terms of the suspension stroke, body acceleration, and tire dynamic load under random road excitation. Table 2 presents the maximum values reached in body acceleration, suspension deflection, and tire deflection with the estimated RMS errors. The results suggest that the proposed robust predictive controller has better performance than the passive suspension system discussed in [21] when the system is subject to sensor faults and external disturbances. In addition, the proposed FTPC structure has the ability to improve the ride quality and road-holding performance under different types of active suspension constraints and in the presense of external disturbances and sensor failures.

Table 2. RMS comparison of the vehicle's dynamic responses with sensor faults.

\begin{tabular}{ccccccc}
\hline Suspension Types & \multicolumn{2}{c}{$\begin{array}{c}\text { Body Acceleration } \\
\left(\mathbf{m} / \mathbf{s}^{2}\right)\end{array}$} & \multicolumn{2}{c}{$\begin{array}{c}\text { Suspension } \\
\text { Deflection } \mathbf{( m )}\end{array}$} & \multicolumn{2}{c}{ Tire Deflection (m) } \\
\hline & Max & RMS & Max & RMS & Max & RMS \\
Passive & 8.695 & 2.432 & 0.0450 & 0.0102 & 0.058 & $3.452 \times 10^{-3}$ \\
Active FTPC & 6.3232 & 1.317 & 0.0200 & 0.0066 & 0.0025 & $4.7913 \times 10^{-4}$ \\
\hline
\end{tabular}

Note that the illustrated performance of the proposed fault predictive controller is due to the following:

The proposed predictive control structure is well adapted for LPV models such as LPV representations for automotive suspension systems. In addition, the external disturbances and faults in the output channel are already considered in the predictive control design procedures, which means that the controller is designed to meet both the stability of the vertical motion of the car's body and the passenger's isolation from vibrations caused by rough roads.

The proposed predictive controller has the ability to anticipate any errors caused by external disturbances and sensor failures, which eventually helps to reduce the impact of these errors while upper limits are imposed on the dynamic force of the motor. Note that the upper limits are used to avoid discomfort to passengers and damage to the vehicle's components.

\section{Conclusions}

In this work, an observer-based robust fault-tolerant predictive control (ORFTPC) approach is proposed for quarter-car active suspension systems with input constraints subject to sensor faults and disturbances. The proposed approach uses two observers: 
a virtual observer is used to reduce the impact of disturbances and sensor failures, and a real observer is employed to obtain the various states and faults.

In the proposed approach, a Linear Parameter-Varying (LPV) model is used to describe the nonlinear quarter-car active suspension system and to develop the proposed controller. Based on the LPV model, an augmented virtual observer was first proposed to improve the estimation of the system's states, while a real observer was designed based on the parameters of the virtual observer to provide a better estimation of both the system's states and sensor faults without the use of a fault isolation module. Furthermore, the stability conditions were derived to ensure the robust stability of the overall closed-loop system. In addition, both the observer gain and the fault-tolerant controller gain were obtained by solving a set of linear matrix inequality constraints. The proposed observer-based controller design was tested on a nonlinear quarter-car active suspension system, and the results demonstrate its ability to improve the vehicle's ride comfort in terms of vibration elimination and fault tolerance. Furthermore, the proposed approach showed its ability to handle faults and external disturbances while satisfying the applied hard constraints, such as a suspension deflection limitation and actuator saturation. In conclusion, the simulation results under external disturbances and sensor faults show that the proposed control method provides improved robustness and performance.

Author Contributions: Conceptualization, A.A., S.B. and I.B.; methodology, A.A., S.B. and M.C.; validation, A.A., S.B., I.B. and M.C; formal analysis, A.A., S.B., I.B. and M.C.; investigation, A.A., S.B., I.B. and M.C.; writing-original draft preparation, S.B., I.B., B.N. and M.C.; writing-review and editing S.B., I.B., B.N. and M.C. All authors have read and agreed to the published version of the manuscript.

Funding: This research received no external funding.

Institutional Review Board Statement: Not applicable.

Informed Consent Statement: Not applicable.

Data Availability Statement: Not applicable.

Conflicts of Interest: The authors declare no conflict of interest.

\section{References}

1. Nazari, R.; Seron, M.M.; De Doná, J.A. Actuator fault tolerant control of systems with polytopic uncertainties using set-based diagnosis and virtual-actuator-based reconfiguration. Automatica 2017, 75, 182-190. [CrossRef]

2. Amin, A.A.; Hasan, K.M. A review of Fault Tolerant Control Systems: Advancements and applications. Measurement 2019, $143,58-68$. [CrossRef]

3. Rotondo, D.; Cristofaro, A.; Johansen, T.A. Fault tolerant control of uncertain dynamical systems using interval virtual actuators. Int. J. Robust. Nonlinear Control 2017, 28, 1-14. [CrossRef]

4. Li, N.; Sun, H.; Zhang, Q. Robust passive adaptive fault tolerant control for stochastic wing flutter via delay control. Eur. J. Control 2019, 48, 74-82. [CrossRef]

5. Fleps-Dezasse, M.; Svaricek, F.; Brembeck, J. Design and experimental assessment of an active fault-tolerant LPV vertical dynamics controller. IEEE Trans. Control Syst. Technol. 2019, 27, 1267-1274. [CrossRef]

6. Lunze, J.; Steffen, T. Control reconfiguration after actuator failures using disturbance decoupling methods. IEEE Trans. Autom. Control 2006, 51, 1590-1601. [CrossRef]

7. Jiang, J.; Yu, X. Fault-tolerant control systems: A comparative study between active and passive approaches. Annu. Rev. Control 2012, 36, 60-72. [CrossRef]

8. Li, X.; Zhu, F. Simultaneous actuator and sensor fault estimation for descriptor LPV system based on H $\infty$ reduced-order observer. Optim. Control Appl. Meth. 2015, 37, 1122-1138. [CrossRef]

9. Rodrigues, M.; Sahnoun, M.; Theilliol, D.; Ponsart, J.-C. Sensor fault detection and isolation filter for polytopic LPV systems: A winding machine application. J. Process Control 2013, 23, 805-816. [CrossRef]

10. Quadros, M.M.; de Bessa, I.V.; Leite, V.J.S.; Palhares, R.M. Fault tolerant control for linear parameter varying systems: An improved robust virtual actuator and sensor approach. ISA Trans. 2020, 104, 356-369. [CrossRef]

11. Li, F.; Zhang, X. Delay-range-dependent robust h1 filtering for singular LPV systems with time variant delay. Int. J. Innov. Comput. Inf. Control 2013, 9, 339-353.

12. Hamdi, H.; Rodrigues, M.; Mechmeche, C.; Theilliol, D.; Braiek, N.B. Fault detection and isolation in linear parameter-varying descriptor systems via proportional integral observer. Int. J. Adapt. Control Signal Process. 2012, 26, 224-240. [CrossRef] 
13. Wang, H.; Daley, S. Actuator fault diagnosis: An adaptive observer-based technique. IEEE Trans. Autom. Control 1996, 41, 1073-1078. [CrossRef]

14. De Lira, S.; Puig, V.; Quevedo, J.; Husar, A. LPV observer design for PEM fuel cell system: Application to fault detection. J. Power Sources 2011, 196, 4298-4305. [CrossRef]

15. Yadegar, Y.; Meskin, N.; Afshar, A. Fault-tolerant control of linear systems using adaptive virtual actuator. Int. J. Control 2019, 92, 1729-1741. [CrossRef]

16. Lee, T.H.; Lim, C.P.; Nahavandi, S.; Roberts, R.G. Observer-Based Ho Fault-Tolerant Control for Linear Systems With Sensor and Actuator Faults. IEEE Syst. J. 2019, 13, 1981-1990. [CrossRef]

17. Liang, X.; Wanga, Q.; Hub, C.; Dong, C. Observer-based Hœfault-tolerant attitude control for satellite with actuator and sensor faults. Aerosp. Sci. Technol. 2019, 95, 105424. [CrossRef]

18. Zahaf, A.; Bououden, S.; Chadli, M.; Chemachema, M. Robust fault tolerant optimal predictive control of hybrid actuators with time varying delay for industrial robot arm. Asian J. Control. 2021. [CrossRef]

19. Tabatabaeipour, S.M.; Stoustrup, J.; Bak, T. Fault-tolerant control of discretetime LPV systems using virtual actuators and sensors. Int. J. Robust. Nonlinear Control 2015, 25, 707-734. [CrossRef]

20. Rotondo, D.; Nejjari, F.; Puig, V. A virtual actuator and sensor approach for fault tolerant control of LPV systems. J. Process Control 2014, 24, 203-222. [CrossRef]

21. Bououden, S.; Chadli, M.; Zhang, L.; Yang, T. Constrained model predictive control for time-varying delay systems: Application to an active car suspension. Int. J. Control Autom. Syst. 2016, 14, 51-58. [CrossRef]

22. Keller, R.; Ding, S.X.; Müller, M.; Stolten, D. Fault-tolerant model predictive control of a direct methanol-fuel cell system with actuator faults. Control Eng. Pract. 2017, 66, 99-115. [CrossRef]

23. Cavanini, L.; Ippoliti, G. Fault tolerant model predictive control for an over-actuated vessel. Ocean Eng. 2018, 160, 1-9. [CrossRef]

24. Zou, T.; Wu, S.; Zhang, R. Improved state space model predictive fault-tolerant control for injection molding batch processes with partial actuator faults using GA optimization. ISA Trans. 2018, 73, 147-153. [CrossRef]

25. Zarch, M.G.; Puig, V.; Poshtan, J.; Shoorehdeli, M.A. Actuator fault tolerance evaluation approach of nonlinear model predictive control systems using viability theory. J. Process Control 2018, 71, 35-45. [CrossRef]

26. Shi, H.; Li, P.; Su, C.; Wang, Y.; Yu, J.; Cao, J. Robust constrained model predictive fault-tolerant control for industrial processes with partial actuator failures and interval time-varying delays. J. Process Control 2019, 75, 187-203. [CrossRef]

27. Sheikhbahaei, R.; Alasty, A.; Vossoughi, G. Robust fault tolerant explicit model predictive control. Automatica 2018, 97, 248-253. [CrossRef]

28. Zhang, R.; Lu, J.; Qu, H.; Gao, F. State space model predictive fault-tolerant control for batch processes with partial actuator failure. J. Process Control 2014, 24, 613-620. [CrossRef]

29. Boyd, S.; Ghaoui, L.; Feron, E.; Balakrishnan, V. Linear Matrix Inequalities in System and Control Theory; Society for Industrial and Applied Mathematics (SIAM): Philadelphia, PA, USA, 1994.

30. Bououden, S.; Chadli, M. Chapter. Predictive Control for Time-Delay Systems: Theory and Applications; Control Strategy for Time-Delay Systems-Part II; Elsevier Academic Press: Amsterdam, The Netherlands, 2021.

31. Bououden, S.; Chadli, M.; Karimi, H.R. A Robust Predictive Control Design for Nonlinear Active Car Suspension Systems. Asian J. Control 2016, 18, 122-132.

32. Shaqarin, T. Robustness analysis of feedback linearisation and LQR control on quarter-car model with cubic nonlinearity. Int. J. Veh. Noise Vib. 2018, 14, 238-250. [CrossRef] 